\title{
Improved automated vessel segmentation for diagnosing eye diseases using fundus images
}

\author{
Sinan Onal ${ }^{* 1}$, Humeyra Dabil-Karacal ${ }^{2}$ \\ ${ }^{1}$ Department of Mechanical and Industrial Engineering, Southern Illinois University, Edwardsville, USA \\ ${ }^{2}$ Ophthalmology and Visual Sciences, School of Medicine, Washington University, St. Louis, USA
}

Received: September 27, 2015

Accepted: November 19, $2015 \quad$ Online Published: November 30, 2015

DOI: $10.5430 / j b g c . v 6 n 1 \mathrm{p} 23$

URL: http://dx.doi.org/10.5430/jbgc.v6n1p23

\begin{abstract}
The retina can provide evidence of diseases originating in other parts of the body. Among the eye diseases that can be diagnosed through a retinal examination, age-related macular degeneration, glaucoma, and diabetic retinopathy are the most common, and they cause vision loss. Although these diseases can be diagnosed by examining blood vessels and the optic disk in retinal images, assessment of blood vessels on colored fundus images is a time-consuming and subjective process. Here, we present an automated blood vessel segmentation algorithm that facilitates the evaluation of diabetic retinopathy through assessment of blood vessel abnormalities. The blood vessels are extracted using a random forest classification model combined with wavelet features and local binary pattern texture information. Discriminant analysis is modified and used for feature selection to train the proposed classification model. The boundary of the optic disk is identified using low-pass filtering, fuzzy c-means clustering, and template matching so that it may be removed and not confound the segmentation analysis. Validation test results using three publicly available retinal image datasets demonstrated that our proposed method achieves as good or better blood vessel segmentation accuracy than the other supervised model approaches examined. Results show that the proposed scheme is able to segment the blood vessels and optic disk structures accurately (Accuracy Index) in 95.80\%, 95.20\%, and 97.10\% of the testing Digital Retinal Images for Vessel Extraction (DRIVE), Structured Analysis of the Retina (STARE), and CHASE_DB1 image datasets respectively. The main advantage of our proposed model is that it provides robust and computationally efficient segmentation of blood vessels and the optic disk. The proposed model aims to provide supportive information for cases in which a diagnosis remains unclear following a clinical examination.
\end{abstract}

Key Words: Blood vessel segmentation, Eye diseases, Fundus image, Random forest, Optic disk

\section{INTRODUCTION}

Signs of numerous diseases associated with the eye, brain, or cardiovascular system appear in the retina. The most common diseases that show signs in the retina, i.e., age-related macular degeneration, glaucoma, and diabetic retinopathy, cause vision loss and are a growing public health concern in the United States. ${ }^{[1]}$ Abundant evidence indicates that these diseases can be prevented through an annual eye screening that includes retinal imaging using a color fundus camera. Diameter changes and altered branching angles of blood vessels are important indicators of these eye diseases, and treatment effectiveness depends on the accurate detection of the structure of the blood vessels. ${ }^{[2]}$ Although ocular fundus imaging is the most common technique for monitoring the health status of patients and for documenting the details of the retinal structures, including the blood vessels, macula,

*Correspondence: Sinan Onal; Email: sonal@ siue.edu; Address: Box 1805 Southern Illinois University, Edwardsville, IL 62026, USA. 
and optic disk, the manual detection of blood vessels and the optic disk in the resulting images is time consuming and subjective.

Various approaches have been proposed for segmenting blood vessels on fundus images. Examples of such approaches include machine learning, ${ }^{[3-12]}$ matched filtering, ${ }^{[13,14]}$ vessel tracking, ${ }^{[15,16]}$ mathematical morphology, ${ }^{[17]}$ model approaches, ${ }^{[18,19]}$ and connected operators. ${ }^{[20,21]}$ Machine-learning methods assign one or more groups to pixels in the retinal image, using multiple numeric pixel features to group them. Among these machine-learning methods, classification models utilizing supervised learning are increasingly of interest as they provide accurate and computationally less expensive classification. The pixels are classified according to learning patterns by a supervised model that uses ground truth images of structures manually segmented by experts. Lupascu et al. ${ }^{[5]}$ proposed the featurebased AdaBoost classifier for blood vessel segmentation. This technique incorporates a multi-feature analysis, with features that represent local and geometrical information of the vessels on different scales. An approach proposed by Lili et al. ${ }^{[4]}$ combined radial projection and support vector machine classifiers for vessel segmentation and was applied to images in the Digital Retinal Images for Vessel Extraction (DRIVE) ${ }^{[22]}$ dataset. In their method, adaptive thresholding is applied to normalize the background of the green channel and to trim the optical disk edges, resulting in segmentation of large vessels. To differentiate thin blood vessels from other structures on the image, the thin vessels are identified by wavelets applied at multiple scales using a support vector machine classification approach. An extracted vessel tree is obtained using a tracking method. Marin et al. ${ }^{[6]}$ developed another supervised model for segmenting blood vessels. A nonlinear, multilayer feed forward neural network is used for training and classification, and their proposed model extracts grayscale values and moment-invariant features to represent each pixel in the retinal images. Osareh et al..$^{[7]}$ proposed a fully automated segmentation scheme to identify blood vessels in retinal images. A feature vector for each image pixel is developed using Gabor filters with various scales and orientations selected. The extracted features are then used to group the pixels through a generative Gaussian mixture model and support vector machine-derived classifiers. Staal ${ }^{[10]}$ introduced a new vessel segmentation scheme using k-nearest neighbors classifiers, with image ridges considered a natural variable for separating vessels from other structures. Soares ${ }^{[12]}$ presented a Bayesian model that groups each pixel according to Gabor wavelet features. Additional information about retinal blood vessel segmentation methods can be found in the survey by Fraz et al. ${ }^{[11]}$ A combination of meth- ods for detecting blood vessel skeletons and multidirectional morphological bit plane slicing has been presented to extract blood vessels. ${ }^{[23]}$ However, these combination methods consider only a single classifier, unlike ensemble classification approaches, and the number of features is much larger than the number of samples, which results in less accurate classification results. Additionally, in the segmentation process, most of these algorithms do not include the extraction of the optic nerve head, a region unwanted for segmenting blood vessels.

The optic disk represents the beginning of the optic nerve, and it is the brightest region where blood vessels converge on the fundus image. It is an approximate vertical ellipsis, with an average horizontal dimension of $1.8 \mathrm{~mm} \pm$ $0.2 \mathrm{~mm}$ and vertical height of $1.9 \mathrm{~mm} \pm 0.2 \mathrm{~mm} .{ }^{[24]}$ Because blood vessels converge at the optic nerve head region, the identification of the optic disk boundary is nontrivial, and a number of methods have been proposed to identify this boundary. Chrastek et al.${ }^{[25]}$ proposed a model that removes thick blood vessels from the optic nerve head region and then applies a Hough transform and active contour model to find the boundary. Welfer et al. ${ }^{[26]}$ presented an adaptive morphological operation and watershed transform to find the optic disk boundary in fundus images. The model suggested by Lowell et al. ${ }^{[27]}$ localizes the optic disk using an active contour model to finalize the boundary identification process. However, all these proposed techniques require intensive computations. $^{[28]}$

The aim of this work was to develop an algorithm that addresses the aforementioned limitations, and to build on a previously developed segmentation technique for an automated extraction of blood vessels on a fundus image that we published the preliminary results of in our earlier work. ${ }^{[29]}$ This improved automated blood vessel extraction model that we propose in this paper evaluates the accuracy of the optic disk detection scheme and provides accuracy comparison using overlapping ratio (ORatio) and the mean absolute distance (MAD). In this model, the blood vessels are extracted using a classification scheme with a random forest. After the green channel is extracted from the original color fundus image, image pre-processing is performed to eliminate the image noise while enhancing the contrast. Wavelet- and LBP-based features are extracted from the image after the pre-processing step, and this is followed by a feature selection process to identify blood vessel structure using a random forest classifier. To finalize the segmentation, the boundary of the optic disk is identified and then removed from the classified image to finalize the segmentation of the blood vessels. Therefore, with this current study, we build on our previous work with important methodological additions and 
extensive validation experiments including larger datasets and comparative evaluation against previously presented algorithms.

The proposed automated blood vessel extraction model and the optic disk segmentation model are presented in Section 2. The experimental results, performance evaluation, and comparison with other commonly used methods are presented in Section 3.

\section{Method}

Because the identification of blood vessels and the optic disk in a fundus image is a time consuming and subjective process, there is a need to automatically extract the retinal structure. However, segmenting this structure is a nontrivial task due to the lack of image contrast, signal noise, and reflection along the centerline of blood vessels.

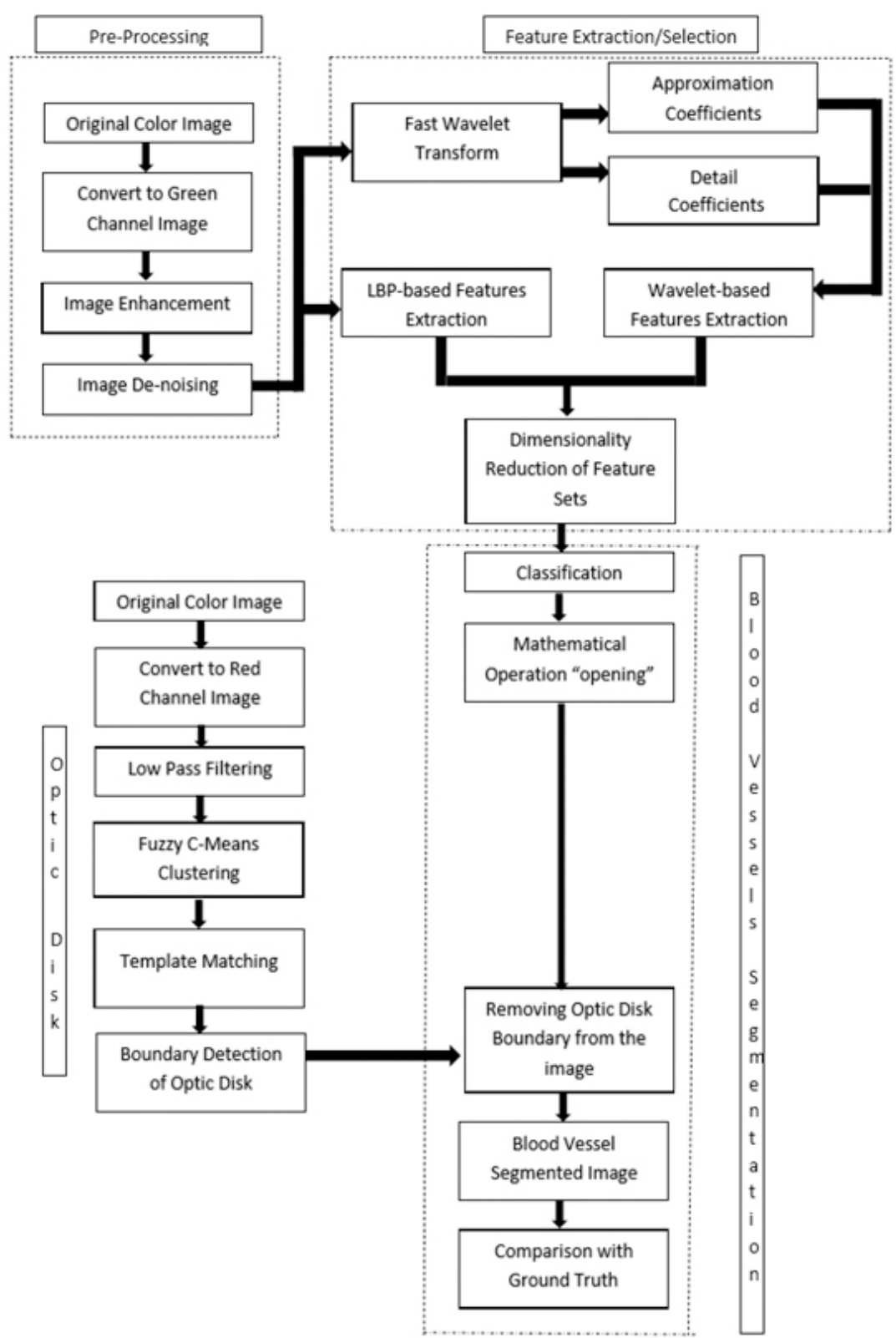

Figure 1. Overview of the proposed segmentation scheme
As shown in Figure 1, our proposed model can be divided into three main phases: pre-processing, feature extraction/selection, and segmentation. A binary mask is generated before applying the proposed model to extract the region of interest, namely, the retinal structure. All the images come with a binary mask that indicate the field of view (FOV) in the DRIVE database. The binary masks have been created for each of the images in the Structured Analysis of the 
Retina (STARE) and CHASE_DB1 databases as explained in Ref. ${ }^{[12]}$ The pre-processing phase begins with the conversion of the original fundus image to the green channel ( 8 bits), which stores pixels with intensity values between 0 and 255 . The next step in the pre-processing phase is noise reduction, followed by contrast enhancement of the images. In the second phase, the wavelet features and LBP texture information are extracted from an image and used to select significant features. In the last phase, a random forest classification model is trained and tested, followed by the optic disk boundary identification and removal processes. Each step in the three main phases of our proposed model is discussed in greater detail below.

\subsection{Image pre-processing}

The first step of the image pre-processing phase is to extract the green channel from the original fundus color image, as the green channel provides better contrast and the most useful information for differentiating blood vessels from the background. ${ }^{[12]}$ The next step, image denoising, incorporates anisotropic diffusion, which can be considered a featurepreserving denoising algorithm. This step aims to remove noise from the image without removing useful information, such as edges, lines, and other details that can be used to interpret the images. However, the effectiveness of anisotropic diffusion image denoising depends on parameters that include a conductance function, an integration constant for numerical stability, and a gradient modulus threshold. We selected the conductance function as $\frac{1}{1+\left(\frac{x}{K}\right)^{2}}$ because it favors larger regions over smaller ones. ${ }^{[30]}$ To select a gradient magnitude threshold, we chose a "noise estimator" that uses the absolute value of an image gradient to estimate the threshold, as described by Canny. ${ }^{[31]}$ Then, the contrast of the denoised image is enhanced by adaptive histogram equalization which combines $6 \times 6$ pixels small regions using bell-shaped histogram to eliminate artificially induced boundaries, as seen in Figure 2.
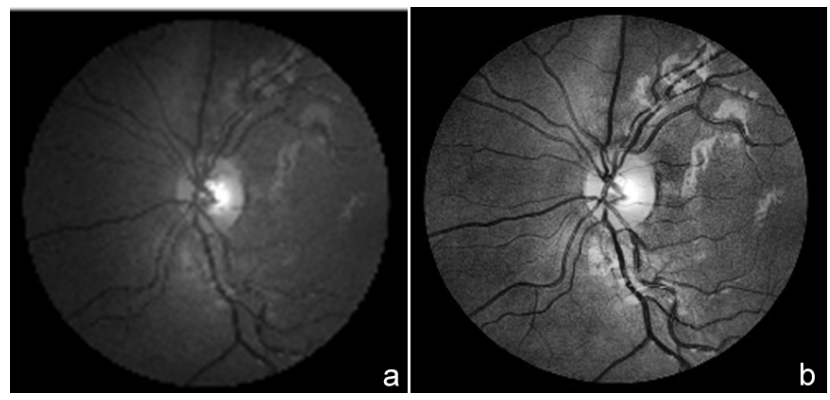

Figure 2. Image pre-processing

(a) Green channel; (b) Image enhancement after anisotropic diffusion

\subsection{Segmentation framework}

\subsubsection{Feature extraction}

The aim of the proposed segmentation scheme is to automatically find blood vessel structures on the image. The vessels are extracted through the grouping of pixels into either blood vessel pixels or background pixels using a supervised model. Feature extraction is a critical step in the classification process. In our proposed model, wavelet features and LBP texture information differentiate the vessel pixels from the background pixels. Texture-based feature vectors contain a numerical metric calculated to provide information about the spatial arrangement of pixel intensities in an image. The feature vector in our model is $f=\left[F_{\text {wavelet }}, F_{L B P}\right]$. The Haar wavelet transform is applied on reference images up to the second level for image decomposition. To extract the wavelet coefficient of each pixel in the image, we used a fast wavelet transform, as this has the ability to discriminate different frequencies and frequent decomposition of an image using scaling and wavelet functions. After applying the fast wavelet transform, the feature vector $F_{\text {wavelet }}$, containing energy, entropy, variance, and standard deviation, is extracted using wavelet coefficients.

We also adapted an $\mathrm{LBP}^{[32]}$ into the classification process because it is an efficient texture operator that identifies patterns of blood vessel features to differentiate them from other structures without contrast deformation. LBP examines the relationship between the central pixel of a cell and its neighbors. A local threshold is used to label each pixel in an image, depending on the intensities of its neighboring pixels, resulting in a new binary number. The examined region is divided into small windows, $3 \times 3$ pixels each. Each pixel located in each window is compared with each of its eight neighbors. If the central pixel's value is greater than the neighbor pixel, the output is assigned as 1 , otherwise it is 0 . This results in an eight-digit binary number. Then, a histogram depicting the frequency of each number is computed over the cell. The histograms of all windows are concatenated to provide the feature vector $F_{L B P}$ for each pixel located at the center of the examined region.

Random forests, a widely used, powerful classification method, are used in our proposed model. However, with randomization of both bagging samples and feature selection, the trees in the forest tend to select uninformative features for node splitting. ${ }^{[33]}$ The resulting algorithm shows poor accuracy when working with high-dimensional data. In addition, a random forest is considered a biased algorithm in feature selection. To overcome these problems, an additional step is incorporated into the classification process so that useful features are selected in learning random forests. In this new step, uninformative or redundant features are removed using 
$p$-value assessment, and a subset of unbiased features is then selected using a modified and adapted discriminant analysis, which derives discriminant subspace from large-scale training data for classification. We have $G$ groups ( $G=2$ pixel groups, i.e., blood vessels and background), and each group has $\mathrm{n}$ samples. The discriminant analysis is described as follows:

$$
\begin{aligned}
& S_{b}=\sum_{i=1}^{G}\left(x_{i}^{J}-x_{i}\right)\left(x_{i}^{J}-x_{i}\right)^{T} \\
& S_{v}=\sum_{i=1}^{G} \sum_{j=1}^{n}\left(x_{i}^{J}-\bar{x}\right)\left(x_{i}^{J}-\bar{x}\right)^{T}
\end{aligned}
$$

where $S_{b}$ is the between-group scatter matrix, and $S_{v}$ is the within-group scatter matrix. The term $x_{i}^{J}$ represents the $j t h$ sample of the $i t h$ group, $x_{i}$ is the mean of the $i t h$ group, and $\bar{x}$ represents the mean of the set. The eigenvalue equation of the discriminant analysis is represented as follows:

$$
S_{b} w=\lambda S_{v} w
$$

Let $w=\left[w_{1} \cdots w_{N}\right]^{T}$ and $x=\left[x_{1} \cdots x_{N}\right]^{T}$ represent the eigenvectors of the covariance matrix for the discriminant analysis and sample, respectively. The discriminant analysis can be used for feature selection as follows:

$$
f=x^{T} w=\sum_{i=1}^{N} x_{i} w_{i}
$$

The first $m$ largest eigenvectors are selected as $E_{1} \cdots E_{m}$. Then, the contribution of each eigenvector is evaluated by

$$
t_{j}=\sum_{i=1}^{m}\left|E_{p j}\right|
$$

where $E_{p j}$ represents the $j t h$ element of vector. The final set of significant features for training the classifier is selected using gained $t_{j}$ by a leave-one-out cross-validation since the relatively small dataset size. In this leave-one-out cross-validation, the model is repeatedly refit leaving out a single observation and then used to derive a prediction for the left-out observation using a mean squared error that minimizes the mean criterion value. This process continues until the addition of more features does not further decrease the criterion.

Published by Sciedu Press

\subsubsection{Classification}

The proposed classification model has been used to differentiate pixels into a blood vessel area or the background region. This is a two-class problem and involves two steps: classifier construction and prediction. The structure of the classifier is constructed using a random forest, as overfitting is not a problem, and it is not very sensitive to outliers in training data. A random forest is an ensemble classifier using multiple decision tree models with additional randomness to bagging. Different subsets of training data are selected with replacements to train each tree, and a randomly selected subset of features is used to split each node. ${ }^{[34]}$ This strategy provides better classification accuracy compared with others. Another advantage of using the random forest is that there are only two parameters that need to be defined: the number of trees in the forest and the depth of the trees at each split.

The classifier is trained using 29 cases at random with replacements to build a subset of data. The subset size is approximately half of the total dataset. Then at each node, 10 numbers of features are selected randomly from the feature set. The feature variable providing the best split is used to conduct a binary split on that node. At the next node, additional 2 features are selected randomly from the feature set, and the process is repeated. The value for $p$ should be much smaller than the total number in the feature set, as suggested in Ref. ${ }^{[34]}$ After the classifier is trained, it is run through all of the trees, and the classification result is accepted as a voting majority of each tree. The anticipated outcome at the end of this process is a set of two groups of pixels that are automatically classified as blood vessels (white) or background (black).

Our segmentation scheme evaluates each pixel independently using a random forest classification model. Because the classification model may produce errors, a correction step is necessary to eliminate the misclassified pixels. Therefore, small regions surrounding the vessel structure are eliminated using a morphological opening operator using "diamond" structuring elements which has a radius of 2 .

\subsubsection{Identification of the optic disk}

The optic disk represents the optic nerve head, and it is the brightest region in the retinal fundus image where blood vessels converge. Optic disk removal is performed before finalizing the segmentation of the blood vessels because the boundary of the optic nerve head is usually undesirable for blood vessel segmentation. Detection of the optic disk is also important to identify abnormalities in the eyes.

Given that the optic disk is clearly visible using the red channel of the fundus image — shown in Figure 3(a) — the disk can be automatically identified using pixel intensity values. 
Low-pass filtering with a filter size of $10 \times 10$ is performed first to remove blood vessels from the optic disk region, as shown in Figure 3(b). Then, the fuzzy c-means (FCM) algorithm is used to group image pixels into two clusters, with every pixel in the image belonging to either optic disk clusters or background clusters, as shown in Figure 3(d). ${ }^{[35]}$ The main advantage of FCM is that it is independent of the training data. Features with close similarities in an image are grouped into the same clusters. The similarity can be defined as the distance from the feature vector to the center of the cluster. The FCM-dependent clustering starts with an initial guess for the cluster center. Then, assigned data points (pixel intensity, in this case) are updated iteratively to move the cluster center to the correct location. Iterations are performed using an identified objective function that minimizes the Euclidian distance between each data point and each cluster center. Because the clustering may result in misclassified regions inside the optic nerve head structure, this region is filled using morphological filling operation based on 4-connected neighborhood connectivity criteria.

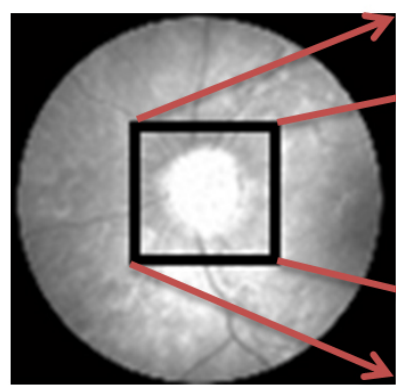

(a)
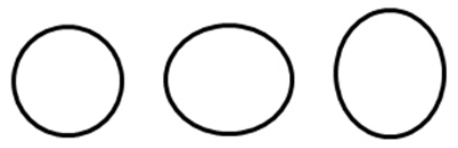

(c)

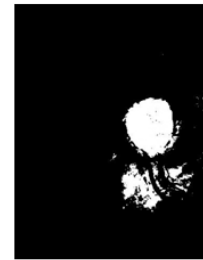

(d)

(e)

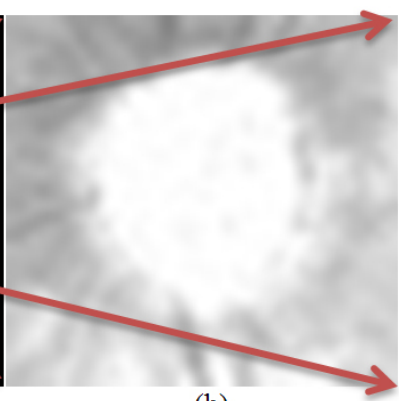

(b)
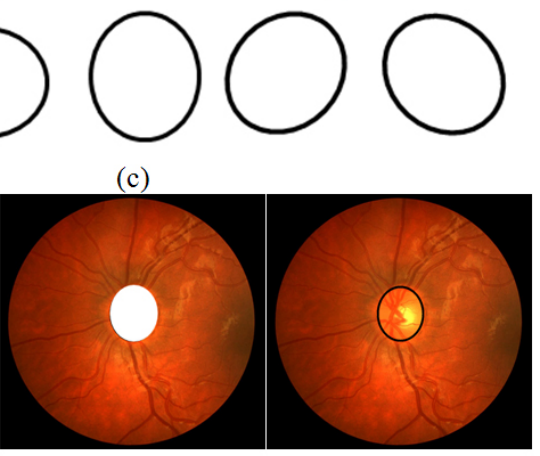

(f)
Figure 3. Optic disk identification

(a) Red channel of fundus image; (b) Low-pass filtering of ROI; (c) One set of the templates used; (d) After fuzzy c-means; (e) Template matching in the region of the optic nerve; and ( $f$ ) Boundary of the optic disk

After grouping the image pixels as either background or optic disk, the next step is to use circular and elliptical templates to determine the optic disk boundary. The diameters ranges from $18 \mathrm{~mm}$ to $19 \mathrm{~mm}$ in $0.2 \mathrm{~mm}$ increments are set up for the circular templates while horizontal lengths from $18 \mathrm{~mm}$ to $19 \mathrm{~mm}$ in $0.2 \mathrm{~mm}$ increments and vertical length $-19 \mathrm{~mm}$ fixed and orientations $\left(0^{\circ}-45^{\circ}-90^{\circ}\right.$ - and $\left.135^{\circ}\right)$ are setup for the elliptical templates in the matching process. An example of the template is shown in Figure 3(c). Matching is performed between the classified image from the red channel, Figure 3(d), and the templates, the inside of the circles and ellipses shown in Figure 3(c). The correlation coefficient is considered an indicator of the match between the template and classified images, the resulting FCM classification. The match with the highest correlation value is selected as the optic nerve region, as shown in Figure 3(e-f).

After finding the region of optic nerve head, the boundary is identified by removing the inside pixels in the region. The boundary is then subtracted from the image that was found using the random forest classifier and morphological opening operations using "diamond" structuring elements which has a radius of 3 to finalize the segmentation process, as shown in Figure 4.

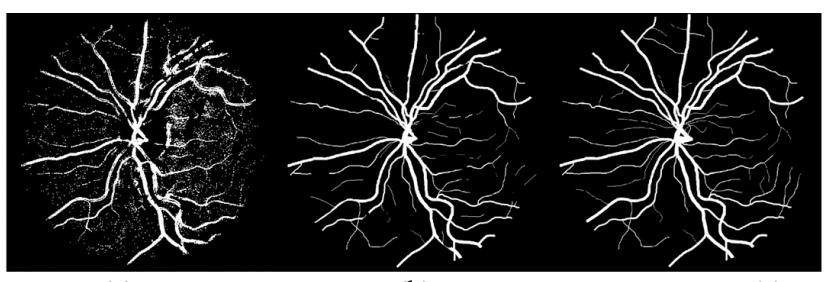

(a)

(b)

(c)

Figure 4. Proposed segmentation scheme using the retinal image dataset CHASE_DB1

(a) Segmentation after random forest classification; (b) Final blood vessel segmentation; and (c) Ground truth of the original image

\section{RESULTS}

\subsection{Image dataset descriptions}

The proposed blood vessel segmentation and optic disk identification schemes were tested using three publicly available retinal image databases, DRIVE, ${ }^{[22]}$ STARE, ${ }^{[36]}$ and CHASE_DB1 (Child Heart and Health Study in England). [37] A total of 40 color fundus images in DRIVE were obtained from a diabetic retinopathy-screening program in the Netherlands. The images were captured using a Canon CRS nonmydriatic 3-CCD camera with a $45^{\circ}$ FOV and a resolution of $768 \times 584$ pixels. Trained researchers manually segmented each fundus image. The STARE database contains 20 fundus images captured with a TopCon TRV-50 fundus camera at a $35^{\circ} \mathrm{FOV}$ and a resolution of $605 \times$ 700 pixels. A total of 28 retinal images in the CHASE_DB1 database were recorded using a Nidek NM-200-D fundus camera. The images were captured at a $30^{\circ} \mathrm{FOV}$ with a resolution of $1,280 \times 960$ pixels. 


\subsection{Evaluation experiments and statistical analysis}

The validation of our proposed blood vessel segmentation method was performed on a representative dataset of these 88 color fundus images. Our proposed model was built using 13 images from the DRIVE dataset, 7 images from the STARE dataset, and 9 images from the CHASE_DB1 dataset and tested using the rest of image dataset (27 DRIVE images, 13 STARE images, and 19 CHASE_DB1 images). The images in the training set are manually segmented by one expert while the images in the testing set are segmented by two other experts. Each image in the STARE and CHASE_DB1 datasets is manually segmented by two experts. However, we measure the performance of our model by comparing with the binary ground truth images provided by the first experts in each dataset.

The performance of the proposed optic disk segmentation method was measured by quantifying the region overlap between the manual and automated segmentations using the ORatio and the MAD. As a benchmark, we compared our model with alternative models proposed by Zeng et al., ${ }^{[38]}$ Boykov et al., ${ }^{[39]}$ and Salazar-Gonzales et al. ${ }^{[28]}$ Unfortunately, it was not possible to test our method against a larger number of alternative methods because most of the published methods did not use a unique benchmark to measure the results of the optic disk segmentation, making a comparison of results difficult. Thus, comparisons of our proposed model were conducted using the topology cut, graph cut, compensation factor, and Markov random field (MRF) image reconstruction. Since manual segmentations of optic disk are not available on STARE and CHASE_DB1 datasets, all methods were compared on the same dataset (DRIVE) only using 27 fundus retinal images. For comparisons with the published methods, all values were derived from the respective manuscripts.

A good segmentation has a high ORatio and a low MAD. According to Niemeijer, ${ }^{[0]}$ an Oratio greater than 0.5000 indicates a successful segmentation. A comparison of the segmentation results using the various models and the DRIVE dataset determined that our proposed optic disk segmentation algorithm provided the second highest average ORatio (0.7840), with an average MAD (5.12) (see Table 1).

The effectiveness of the proposed blood vessel segmentation model was also investigated using receiver operating characteristic (ROC) curves. A ROC curve illustrates the performance of a model by providing the true positive rate versus the false positive rate. In the performance evaluation process, a true positive indicates that the data are classified as "blood vessel" by both the manual and the proposed automated method. Similarly, a true negative is the outcome when data are classified as "background" by both the manual and automated processes. False positives and false negatives were also determined. In addition to ROC curves, accuracy was measured as a performance evaluator using the ratio of the number of correctly classified blood vessel pixels to the number of total pixels in the image.

Table 1. Performance of optic disk segmentation model using the drive dataset

\begin{tabular}{lll}
\hline Methodology & Average ORatio & Average MAD \\
\hline Proposed model & 0.7840 & 5.12 \\
Topology cut $^{[38]}$ & 0.5591 & 10.24 \\
Graph cut $^{[39]}$ & 0.5532 & 9.97 \\
MRF image reconstruction $^{[28]}$ & 0.8240 & 3.39 \\
Compensation factor $^{[28]}$ & 0.7090 & 6.48 \\
\hline
\end{tabular}

We selected a training set to build the proposed classifier. The training dataset was trained using 13 images in the DRIVE dataset, 7 images in the STARE dataset, and 9 images in the CHASE_DB1 dataset. The validation of the proposed model was performed with an image data set of 27 DRIVE, 13 STARE, and 19 CHASE_DB1 fundus images. Ten-fold cross-validation experiments were conducted to evaluate the performance of the proposed model. To find the best parameters, forest size and tree depth, for the random forest classifier, we conducted 100 runs of leave-one-out crossvalidation experiments with a grid search. The best result was obtained with a forest size of 8 and a tree depth of 5 .

Table 2 provides a comparison of the sensitivity (Sen), specificity (Spe), accuracy (Acc), and area under the ROC curve for the proposed model with the other available supervised blood vessel segmentation methods. Accuracy comparisons were performed using values from the respective manuscripts. We found that our proposed model provided better segmentation results than the alternative methods using the DRIVE dataset. The average sensitivity and specificity of the proposed model were $81.38 \%$ and $97.40 \%$, respectively. Our proposed model showed the highest accuracy $(95.80 \%)$ and ROC curve value (97.14\%) among the compared models.

The proposed model was also evaluated using the STARE image dataset. The accuracy of our proposed model was $95.20 \%$. Although this was the second highest value, the differences in accuracy among all the models were small. Sensitivity and specificity of the proposed model were $72.50 \%$ and $98.08 \%$ respectively, with the highest sensitivity provided by You et al. ${ }^{[8]}(72.60 \%)$, and the highest specificity by Marin et al. $(98.19 \%)$.

The table also compares the performance of our proposed model for automated segmentation of blood vessels with that suggested by Fraz et al. ${ }^{[3]}$ using the CHASE_DB1 dataset. 
The average accuracy of our proposed model was $97.10 \%$, which was more accurate, and the sensitivity and specificity of the proposed model were also slightly higher than those for the model published by Fraz et al.

Table 2. Performance of blood vessel segmentation models using different image datasets

\begin{tabular}{|c|c|c|c|c|c|}
\hline Methodology & Dataset & Sen & Spe & Acc & ROC \\
\hline \multirow{3}{*}{$\begin{array}{l}\text { Proposed } \\
\text { model }\end{array}$} & DRIVE & 0.8138 & 0.9740 & 0.9580 & 0.9714 \\
\hline & STARE & 0.7250 & 0.9808 & 0.9520 & 0.9620 \\
\hline & CHASE_DB1 & 0.7516 & 0.9748 & 0.9710 & 0.9814 \\
\hline \multirow{3}{*}{ Onal (2015) } & DRIVE & 0.7980 & 0.9850 & 0.9484 & 0.9674 \\
\hline & STARE & 0.7190 & 0.9760 & 0.9519 & 0.9590 \\
\hline & CHASE_DB1 & - & - & - & - \\
\hline \multirow{3}{*}{ Staal et al. ${ }^{[10]}$} & DRIVE & - & - & 0.9442 & 0.9520 \\
\hline & STARE & - & - & 0.9516 & 0.9614 \\
\hline & CHASE_DB1 & - & - & - & - \\
\hline \multirow{3}{*}{$\begin{array}{l}\text { Soares et } \\
\text { al. }{ }^{[12]}\end{array}$} & DRIVE & - & - & 0.9466 & 0.9614 \\
\hline & STARE & - & - & 0.9480 & 0.9671 \\
\hline & CHASE_DB1 & - & - & - & - \\
\hline \multirow{3}{*}{ Lili et al. ${ }^{[4]}$} & DRIVE & 0.7760 & - & 0.9328 & - \\
\hline & STARE & - & - & - & - \\
\hline & CHASE_DB1 & - & - & - & - \\
\hline \multirow{3}{*}{ You et al. ${ }^{[8]}$} & DRIVE & 0.7410 & 0.9751 & 0.9434 & - \\
\hline & STARE & 0.7260 & 0.9756 & 0.9497 & - \\
\hline & CHASE_DB1 & - & - & - & - \\
\hline \multirow{3}{*}{ Marin et al. ${ }^{[6]}$} & DRIVE & 0.7067 & 0.9801 & 0.9452 & 0.9588 \\
\hline & STARE & 0.6944 & 0.9819 & 0.9526 & 0.9769 \\
\hline & CHASE_DB1 & & & & \\
\hline \multirow{3}{*}{$\begin{array}{l}\text { Niemeijer et } \\
\text { al. }{ }^{[22]}\end{array}$} & DRIVE & 0.7145 & - & 0.9416 & 0.9294 \\
\hline & STARE & - & - & - & - \\
\hline & CHASE_DB1 & - & - & - & - \\
\hline \multirow{3}{*}{ Fraz et al. ${ }^{[3]}$} & DRIVE & - & - & - & - \\
\hline & STARE & - & - & - & - \\
\hline & CHASE_DB1 & 0.7224 & 0.9711 & 0.9469 & 0.9712 \\
\hline \multirow{3}{*}{$\begin{array}{l}\text { Salazar et } \\
\text { al. }{ }^{[36]}\end{array}$} & DRIVE & - & - & 0.9412 & - \\
\hline & STARE & - & - & 0.9441 & - \\
\hline & CHASE_DB1 & - & - & - & - \\
\hline
\end{tabular}

The sensitivity of our proposed model with various settings was also analyzed, and the results are shown in Table 3. Classification accuracy was improved after the morphological operations and the optic disk removal process. Each method was tested against a manually segmented region identified by experts. The random forest classification method without the morphological operations and the optic disk removal was faster than our proposed method. However, the segmentation accuracies of this classification method using images from all three databases were lower than those for our proposed method. As shown in Table 3, incorporating the optic disk removal step increased the accuracy of the blood vessel seg- mentation process by approximately $1.5 \%$ in all three image datasets.

Table 3. Accuracy of our proposed model with various settings

\begin{tabular}{llllll}
\hline Methodology & Database & Sen & Spe & Acc & ROC \\
\hline V1: Random & DRIVE & 0.7418 & 0.9114 & 0.8952 & 0.9234 \\
forest & STARE & 0.6784 & 0.9228 & 0.8719 & 0.9348 \\
classification & CHASE_DB1 & 0.6986 & 0.9216 & 0.9004 & 0.9288 \\
V2: Version 1 and & DRIVE & 0.7622 & 0.9392 & 0.9408 & 0.9566 \\
morphological & STARE & 0.7042 & 0.9368 & 0.9402 & 0.9444 \\
operations & CHASE_DB1 & 0.7246 & 0.9468 & 0.9524 & 0.9516 \\
\multirow{2}{*}{ V3: Version 2 and } & DRIVE & 0.8138 & 0.9740 & 0.9580 & 0.9714 \\
optic disk removal & STARE & 0.7250 & 0.9808 & 0.9520 & 0.9620 \\
& CHASE_DB1 & 0.7516 & 0.9748 & 0.9710 & 0.9814 \\
\hline
\end{tabular}

A statistical significant test using DeLong's test ${ }^{[41]}$ has also been conducted to assess the significance of adding each component. The result of pairwise comparison of all ROC curves is presented in Table 4: the difference between the areas, the standard error, the $95 \%$ confidence interval for the difference and $p$-value. If $p$ is less than the conventional $5 \%$ $(p<.05)$, the conclusion is that the each two compared areas are significantly different.

\section{Discussion}

In this study, we presented a new blood vessel and optic disk segmentation approach that integrates wavelet- and LBP-based texture information to extract blood vessels from a colored fundus image using a random forest supervised learning model. The main contribution of this model is that it integrates an ensemble classification approach with discriminant analysis using feature selection. An optic disk removal process is also incorporated into the segmentation process to increase the accuracy of the proposed segmentation scheme. The blood vessel structure is identified using wavelet features and LBP local information, which examines the relationship between the central pixel of a cell and its neighbors. Discriminant analysis is modified and used for significant features selection to train the classification model. The optic disk removal process is performed before finalizing the blood vessel segmentation, as the edges of the optic disk are undesirable during blood vessel segmentation. Previously presented optic disk removal scheme has been improved in this study by adding a template matching algorithm to increase the accuracy of the blood vessel segmentation. Compared with other segmentation approaches, our proposed method achieves as good or higher blood vessel segmentation accuracy. Optic disk removal process improves the segmentation performance by approximately $1.5 \%$ in all three image datasets. 
Table 4. Pairwise comparison of ROC curves

\begin{tabular}{|c|c|c|c|c|c|}
\hline \multicolumn{6}{|l|}{ DRIVE } \\
\hline $\mathrm{V} 3 \sim \mathrm{V} 2$ & & V3 V1 & & $\mathrm{V} 2 \sim \mathrm{V} 1$ & \\
\hline Difference between areas & 0.0158 & Difference between areas & 0.0480 & Difference between areas & 0.0322 \\
\hline Standard error & 0.0482 & Standard error & 0.0363 & Standard error & 0.0500 \\
\hline 95\% confidence interval & $\begin{array}{l}0.165 \text { to } \\
0.369\end{array}$ & 95\% confidence interval & $\begin{array}{l}0.012 \text { to } \\
0.225\end{array}$ & 95\% confidence interval & $\begin{array}{l}-0.011 \text { to } \\
0.062\end{array}$ \\
\hline Significance level & $p=.038$ & Significance level & $p=.002$ & Significance level & $p=.460$ \\
\hline \multicolumn{6}{|l|}{ STARE } \\
\hline $\mathrm{V} 3 \sim \mathrm{V} 2$ & & $\mathrm{~V} 3 \sim \mathrm{V} 1$ & & $\mathrm{~V} 2 \sim \mathrm{V} 1$ & \\
\hline Difference between areas & 0.0176 & Difference between areas & 0.0272 & Difference between areas & 0.0096 \\
\hline Standard error & 0.0250 & Standard error & 0.0381 & Standard error & 0.0780 \\
\hline 95\% confidence interval & $\begin{array}{l}0.008 \text { to } \\
0.425\end{array}$ & 95\% confidence interval & $\begin{array}{l}0.010 \text { to } \\
0.436\end{array}$ & 95\% confidence interval & $\begin{array}{l}-0.005 \text { to } \\
0.012\end{array}$ \\
\hline Significance level & $p=.033$ & Significance level & $p=.008$ & Significance level & $p=.700$ \\
\hline \multicolumn{6}{|l|}{ CHASE_DB1 } \\
\hline $\mathrm{V} 3 \sim \mathrm{V} 2$ & & V3 V1 & & $\mathrm{V} 2 \sim \mathrm{V} 1$ & \\
\hline Difference between areas & 0.0298 & Difference between areas & 0.0526 & Difference between areas & 0.0228 \\
\hline Standard error & 0.0351 & Standard error & 0.0450 & Standard error & 0.0681 \\
\hline 95\% confidence interval & $\begin{array}{l}0.010 \text { to } \\
0.925\end{array}$ & 95\% confidence interval & $\begin{array}{l}0.043 \text { to } \\
0.198\end{array}$ & 95\% confidence interval & $\begin{array}{l}-0.013 \text { to } \\
0.098\end{array}$ \\
\hline Significance level & $p=.024$ & Significance level & $p=.001$ & Significance level & $p=.540$ \\
\hline
\end{tabular}

For the optic disk segmentation, Table 1 indicates the results of the performance of our proposed model using the DRIVE image dataset compared with those of the other supervised models. Our proposed model using low-pass filtering, FCM clustering, and template matching provided the best performance.

Sensitivity, specificity, accuracy, and ROC curves were used to evaluate the effectiveness of our proposed model. As shown in Table 2, the proposed model provided better sensitivity, specificity, accuracy, and ROC curve values on images from the DRIVE dataset. It was the second most accurate, sensitive, and specific model on images from the STARE dataset and showed the highest accuracy on images from the CHASE_DB1 dataset. Thus, overall, our proposed model provided as good or better accuracy than the other supervised models tested on these datasets. A strong advantage of our proposed model is that it offers a robust and computationally efficient segmentation scheme for blood vessel extraction using multiple datasets without retraining. Other advantages of the model are that overfitting is not a problem, and it is not overly sensitive to outliers in the training data during the classification of the pixels because of the use of random forest classification.

Although the proposed model achieves high accuracy on blood vessel segmentation, there are a few limitations to the model. One drawback is that performance depends on the training datasets; therefore, the model would require a new training dataset before it could be used on new datasets. Another limitation of our segmentation model is that although it has been evaluated using publicly available DRIVE, STARE, and CHASE_DB1 databases, the image resolution in these datasets is limited; thus, better resolution would be required to identify thinner vessels. In addition, these image datasets are small, containing a total of only 88 images. To address both of these limitations, we plan to acquire 200 color fundus images using a better camera with higher resolution. Currently the proposed model can be used as a supportive, objective, and quantitative decision tool for assessment of only those eye diseases involving blood vessels or the optic disk. However, we plan to modify the model to include other significant eye diseases that can be identified and diagnosed using images of the retina. 


\section{REFERENCES}

[1] Abràmoff M, Garvin M, Sonka M. Retinal imaging and image analysis. IEEE Reviews in Biomedical Engineering. 2010; 3: 169-208. http://dx.doi.org/10.1109/RBME. 2010.2084567

[2] Gelman R, Martinez-Perez ME, Vanderveen DK, et al. Diagnosis of Plus Disease in Retinopathy of Prematurity Using Retinal Image Multiscale Analysis. Investigative Ophthalmology \& Visual Science. 2005; 46: 4734-4738. PMid: 16303973. http://dx.doi.org/10. 1167 /iovs. 05-0646

[3] Fraz MM, Remagnino P, Hoppe A, et al. An Ensemble ClassificationBased Approach Applied to Retinal Blood Vessel Segmentation. IEEE Transactions on Biomedical Engineering. 2012; 59: 2538-2548. PMid: 22736688. http://dx.doi.org/10.1109/TBME. 2012.2 205687

[4] Lili X, Shuqian L. A novel method for blood vessel detection from retinal images. Biomed Engineering Online. 2010.

[5] Lupascu CA, Tegolo D, Trucco E. Retinal Vessel Segmentation using AdaBoost. IEEE Transactions on Information Technology in Biomedicine. 2010; 14: 1267-1274. PMid: 20529750. http: //dx.doi.org/10.1109/TITB.2010.2052282

[6] Marin D, Aquino A, Gegundez-Arias ME, et al. A New Supervised Method for Blood Vessel Segmentation in Retinal Images by Using Gray-Level and Moment Invariants-Based Features. IEEE Transactions on Medical Imaging. 2011; 30: 146-158. PMid: 20699207. http://dx.doi.org/10.1109/TMI . 2010.2064333

[7] Osareh A, Shadgar B. Automatic Segmentation of Blood Vessels in Colour Retinal Images using Spatial Gabor Filter and Multiscale Analysis. 13th International Conference on Biomedical Engineering; 2008; 274-276p. Singapore.

[8] You X, Peng Q, Yuan Y, et al. Segmentation of retinal blood vessels using the radial projection and semi-supervised approach. Pattern Recognition. 2011; 44: 2314-2324. http://dx.doi.org/10.10 $16 / j \cdot$ patcog. 2011.01.007

[9] Ng J, Clay ST, Barman SA, et al. Maximum likelihood estimation of vessel parameters from scale space analysis, Image and $\mathrm{Vi}$ sion Computing. Image and Vision Computing. 2010; 28: 55-63. http://dx.doi.org/10.1016/j.imavis.2009.04.019

[10] Staal J, Abramoff MD, Niemeijer M, et al. Ridge-based vessel segmentation in color images of the retina. IEEE Transactions on Medical Imaging. 2004; 23: 501-509. PMid: 15084075. http: //dx.doi.org/10.1109/TMI.2004.825627

[11] Fraz M, Remagnino P, Hoppe A, et al. Blood vessel segmentation methodologies in retinal images - A survey. Computer Methods and Programs in Biomedicine. 2012; 108: 407-433. PMid: 22525589. http://dx.doi.org/10.1016/j.cmpb.2012.03.009

[12] Soares JV, Leandro JJ, Cesar Júnior RM, et al. Retinal vessel segmentation using the 2-D Gabor wavelet and supervised classification. IEEE Transactions on Medical Imaging. 2006; 25: 1214-22. PMid: 16967806. http://dx.doi.org/10.1109/TMI . 2006.879967

[13] Zhang B, Zhang L, Karray F. Retinal vessel extraction by matched filter with first-order derivative of Gaussian. Computers in Biology and Medicine. 2010; 40: 438-445. PMid: 20202631. http: //dx.doi.org/10.1016/j.compbiomed.2010.02.008

[14] Cinsdikici MG, Aydin D. Detection of blood vessels in ophthalmoscope images using MF/ant (matched filter/antcolony) algorithm. Computer Methods and Programs in Biomedicine. 2009; 96: 85-95. PMid: 19419790. http://dx.doi.org/10.1016/j.cmpb. 2009 .04 .005

[15] Kelvin P, Ghassan H, Rafeef A. Live-vessel: extending livewire for simultaneous extraction of optimal medial and boundary paths in vascular images. 10th International Conference on Medical Image Computing and Computer-Assisted Intervention; 2007; Australia.
[16] Delibasis KK, Kechriniotis A, Tsonos C, et al. Automatic modelbased tracing algorithm for vessel segmentation and diameter estimation. Computer Methods and Programs in Biomedicine. 2010; 100: 108-122. PMid: 20363522. http://dx.doi.org/10.1016/j.c mpb.2010.03.004

[17] Sun K, Chen Z, Jiang S, et al. Morphological multiscale enhancement, fuzzy filter and watershed for vascular tree extraction in angiogram. Medical Systems. 2010; 35: 811-824. PMid: 20703728. http://dx.doi.org/10.1007/s10916-010-9466-3

[18] Al-Diri B, Hunter A, Steel D. An active contour model for segmenting and measuring retinal vessels. IEEE Transactions on Medical Imaging. 2009; 28: 1488-1497. PMid: 19336294. http://dx.doi .org/10.1109/TMI . 2009.2017941

[19] Sum KW, Cheung PYS. Vessel extraction under non-uniform illumination: a level set approach. IEEE Transactions on Biomedical Engineering. 2008; 55: 358-360. PMid: 18232383. http: //dx.doi.org/10.1109/TBME. 2007.896587

[20] Xu Y, Geraud T, Najman L. Connected Filtering on Tree-Based Shape-Spaces. IEEE Transactions on Pattern Analysis and Machine Intelligence. 2015; 1-99. http://dx.doi.org/10.1109/tpami .2015.2441070

[21] Perret B, Cousty J, Tankyevych O, et al. Directed Connected Operators: Asymmetric Hierarchies for Image Filtering and Segmentation. IEEE Transactions on Pattern Analysis and Machine Intelligence. 2015; 37: 1162-1176. PMid: 26357340. http://dx.doi.org/10. 1109/TPAMI . 2014. 2366145

[22] Niemeijer M, Staal JJ, Ginneken BV, et al. DRIVE: digital retinal images for vessel extraction. 2004, Nov 15. Available from: http://www.isi.uu.nl/Research/Databases/DRIVE

[23] Fraz MM, Basit A, Barman SA. Application of Morphological Bit Planes in Retinal Blood Vessel Extraction. Digital Imaging. 2013; 26: 274-286. PMid: 22832895. http://dx.doi.org/10.1007/s 10278-012-9513-3

[24] Sinthanayothin C, Boyce JF, Cook HL, et al. Automated localisation of the optic disk, fovea, and retinal blood vessels from digital colour fundus images. British Journal of Ophthalmology. 1999; 83: 902-910. PMid: 10413690. http://dx.doi.org/10.1136/bjo.83.8.90 2

[25] Chrástek R, Wolf M, Donath K, et al. Automated segmentation of the optic nerve head for diagnosis of glaucoma. Medical Image Analysis. 2005; 9: 297-314. PMid: 15950894. http://dx.doi.org/10.10 16/j.media. 2004.12.004

[26] Welfer D, Scharcanski J, Kitamura C, et al. Segmentation of the optic disk in color eye fundus images using an adaptive morphological approach. Computers in Biology and Medicine. 2010; 40: 124-137. PMid: 20045104. http://dx.doi.org/10.1016/j.compbiome d. 2009.11.009

[27] Lowell J, Hunter A, Steel D, et al. Optic nerve head segmentation. IEEE Transactions on Medical Imaging. 2004; 23: 256-264. PMid: 14964569. http://dx.doi.org/10.1109/TMI . 2003. 823261

[28] Salazar-Gonzalez A, Kaba D, Yongmin L, et al. Segmentation of the Blood Vessels and Optic Disk in Retinal Images. IEEE Journal of Biomedical and Health Informatics. 2014; 18: 1874-1886. PMid: 25265617. http://dx.doi.org/10.1109/JBHI. 2014.2 302749

[29] Onal S, Adeshina A, Dabil-Karacal H. A machine Learning Approach to Improve the Diagnosis of Diabetic Retinopathy Using Fundus Image. 2015 IIE- Industrial and Systems Engineering Research Conference; 2015; Nashville, TN.

[30] Perona P, Malik J. Scale-space and edge detection using anisotropic diffusion. IEEE Trans. Pattern Anal. Machine Intell. 1990; 12: 629639. http://dx.doi.org/10.1109/34.56205 
[31] Canny J. Computational Approach To Edge Detection. IEEE Transaction on Pattern Analysis and Machine Intelligence. 1986; 8: 679-698. http://dx.doi .org/10.1109/TPAMI . 1986.4767851

[32] He DC, Wang L. Texture Unit, Texture Spectrum, And Texture Analysis. IEEE Transactions on Geoscience and Remote Sensing. 1990; 28 : 509-512. http://dx.doi.org/10.1109/TGRS.1990.572934

[33] Nguyen TT, Huang JZ, Nguyen TT. Unbiased feature selection in learning random forests for high-dimensional data. ScientificWorldJournal. 2015; 2015: 471371. PMid: 25879059. http://dx.doi.o rg/10.1155/2015/471371

[34] Breiman L. Random Forest. Machine Learning. 2001; 45: 5-32. http://dx.doi.org/10.1023/A : 1010933404324

[35] Bezdek JC. Pattern recognition with fuzzy objective function algorithms. Kluwer Academic Publishers; 1981. http://dx.doi.org /10.1007/978-1-4757-0450-1

[36] Hoover AD, Kouznetsova V, Goldbaum M. Locating blood vessels in retinal images by piecewise threshold probing of a matched filter response. IEEE Transactions on Medical Imaging. 2000; 19: 203-210. PMid: 10875704. http://dx.doi.org/10.1109/42.845178
[37] Retinal Image Database. 2011, May 1. Available from: http: //blogs.kingston.ac.uk/retinal/chasedb1/

[38] Zeng Y, Samaras D, Chen W, et al. Topology cuts: A novel mincut/max-flow algorithm for topology preserving segmentation in N-D images. Computer Vision and Image Understanding. 2008; 112: 8190. http://dx.doi.org/10.1016/j.cviu.2008.07.008

[39] Boykov Y, Funka-Lea G. Graph Cuts and Efficient N-D Image Segmentation. International Journal of Computer Vision. 2006; 70: 109131. http://dx.doi.org/10.1007/s11263-006-7934-5

[40] Niemeijer M, Abr'amoff MD, Ginneken BV. Segmentation of the optic disk, macula and vascular arch in fundus photographs. IEEE Transactions on Medical Imaging. 2007; 26: 116-127. PMid: 17243590 http://dx.doi.org/10.1109/TMI . 2006.885336

[41] DeLong ER, DeLong DM, Clarke-Pearson DL. Comparing the areas under two or more correlated receiver operating characteristic curves: a nonparametric approach. Biometrics. 1988; 44: 837-845. PMid: 3203132. http://dx.doi.org/10.2307/2531595 